\title{
Distributions of Zeros of Solutions for Third-Order Differential Equations with Variable Coefficients
}

\author{
Samir H. Saker and Mohammed A. Arahet \\ Department of Mathematics, Faculty of Science, Mansoura 35516, Egypt \\ Correspondence should be addressed to Mohammed A. Arahet; malroheet@yahoo.com
}

Received 9 November 2014; Accepted 11 December 2014

Academic Editor: Zhen-Lai Han

Copyright (C) 2015 S. H. Saker and M. A. Arahet. This is an open access article distributed under the Creative Commons Attribution License, which permits unrestricted use, distribution, and reproduction in any medium, provided the original work is properly cited.

For the third-order linear differential equations of the form $\left(r(t) x^{\prime \prime}(t)\right)^{\prime}+p(t) x^{\prime}(t)+q(t) x(t)=0$, we will establish lower bounds for the distance between zeros of a solution and/or its derivatives. The main results will be proved by making use of Hardy's inequality and some generalizations of Opial and Wirtinger type inequalities.

\section{Introduction}

The mathematical description of some physical systems demands that we often solve linear differential equations subject to some boundary conditions. Some of these problems are the mathematical models of the deflection of beams. These beams, which appear in many structures, deflect under their own weight or under the influence of some external forces. For example, if a load is applied to the beam in a vertical plane containing the axis of symmetry, the beam undergoes a distortion, and the curve connecting the centroids of all cross sections is called the deflection curve or elastic curve. In elasticity, it is shown that the deflection of the curve, say $y(x)$ measured from the $x$ axis, approximates the shape of the beam and satisfies a linear fourth-order differential equation on an interval, say $[\alpha, \beta]$, with some boundary conditions. The distribution of boundary conditions which is the distribution of zeros of solutions of differential equations has been started by Picard $[1,2]$, who derived some uniqueness results for solutions of the second-order nonlinear differential equation with two-point boundary conditions when the nonlinear function satisfies the Lipschitz condition. The distribution of zeros of $n$ th-order differential equations with more than two points has been considered by Niccoletti [3]. Motivated by the work of Picard and Niccoletti, de la Vallée
Poussin [4] considered the general $n$ th-order linear differential equation

$$
\begin{array}{r}
y^{(n)}(t)+p_{1}(t) y^{(n-1)}(t)+\cdots+p_{n}(t) y(t)=0, \\
t \in \mathbb{Q}=[a, b],
\end{array}
$$

with real coefficients that are locally integrable inside $\llbracket$ and studied the disconjugacy of solutions.

Equation (1) is said to be disconjugate on an interval d if every nontrivial solution has less than $n$ zeros on $\mathbb{\square}$, with multiple zeros being counted according to their multiplicity. More precisely, disconjugate on a connected set means that the number of zeros of a nontrivial solution cannot equal the order of the equation. Equation (1) is said to be $(k, n-k)$-disconjugate on an interval $\llbracket$ if no nontrivial solution has a zero of order $k$ followed by a zero of order $n-k$.

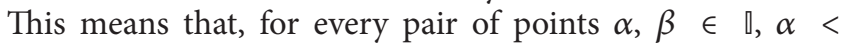
$\beta$, there does not exist a nontrivial solution of (1) which satisfies

$$
\begin{aligned}
& y^{(i)}(\alpha)=0, \quad i=0, \ldots, k-1, \\
& y^{(j)}(\beta)=0, \quad j=0, \ldots, n-k-1 .
\end{aligned}
$$

The last value of $\beta$, such that there exists a nontrivial solution which satisfies (2), is called the $(k, n-k)$-conjugate point of $\alpha$. The first work that has been published by de la Vallée 
Poussin in 1929 was on the evaluation of the length of the interval $[0, h]$ in which the boundary problem $y\left(t_{1}\right)=$ $y\left(t_{2}\right)=\cdots=y\left(t_{n}\right)=0,\left(0 \leq t_{1}<t_{2}<\cdots<t_{n} \leq h\right)$, for the linear differential equation (1) only admits the null solution.

Following the way indicated by de la Vallée Poussin, one has tried to evaluate the length $h$ in a function of the upper bounds of the coefficients. The precise evaluation of the maximal length of the considered interval has only been obtained for second-order differential equations $[5,6]$ with the best possible constant $1 / 4$. Coming then to (1), de la Vallée Poussin proved that this equation is disconjugate in $[0, h]$ if the coefficients and the length of the interval satisfy the inequality

$$
\sum_{i=1}^{n} P_{i} \frac{h^{i}}{i}<1, \quad P_{i}=\max _{a \leq t \leq b}\left|p_{i}(t)\right|, \quad \text { for } i=1,2, \ldots, n
$$

This is the part of his paper which has motivated the many refinements and generalizations described in the analysis. The importance of Poussin's work has been emphasized and testified by many authors in the literature; we refer the reader to the papers [7-16] and the references cited therein. For completeness, we recall some of the related results which motivate the contents of this paper. Lasota [8] considered the third-order differential equation

$$
\begin{array}{r}
y^{\prime \prime \prime}(t)+p_{1}(t) y^{\prime \prime}(t)+p_{2}(t) y^{\prime}(t)+p_{3}(t) y(t)=0, \\
t \in[a, b],
\end{array}
$$

and proved that if

$$
P_{1} \frac{h}{4}+P_{2} \frac{h^{2}}{\pi^{2}}+P_{3} \frac{h^{3}}{2 \pi^{2}} \leq 1
$$

then (4) is disconjugate, where $P_{i}=\max \left|p_{i}(t)\right|$ and $h=b-a$. Mathsen [9] proved that (4) is disconjugate if $p_{2}(x) \leq 0$ on $[a, b]$ and

$$
\left(2 h_{1}+1\right) P_{3} \frac{\left[\exp \left(2 P_{1} h_{1}\right)-\exp \left(P_{1} h_{1}\right)-h_{1} P_{1}\right]}{P_{1}^{2}} \leq 1
$$

where $P_{1}=\max \left|p_{1}(t)\right|, P_{3}=\max \left|p_{3}(t)\right|$ on $[a, b]$, and $h_{1}=$ $(b-a) / 2$. Casadei [7] also proved that if

$$
P_{1} \frac{h}{2}+P_{2} \frac{h^{2}}{8}+P_{3} \frac{h^{3}}{24} \leq 1
$$

then (4) is disconjugate. Agarwal and Krishnamurthy [17] also proved that if

$$
P_{1} \frac{2 h}{3}+P_{2} \frac{h^{2}}{6}+P_{3} \frac{2 h^{3}}{81} \leq 1
$$

then (4) is disconjugate.
Motivated by these papers, we will study the distribution of zeros of the solutions of the third-order differential equation

$$
\left(r(t) x^{\prime \prime}(t)\right)^{\prime}+p(t) x^{\prime}(t)+q(t) x(t)=0, \quad t \in \mathbb{\mathbb { N }}
$$

where $\square$ is an interval of reals and $r(t), p(t)$, and $q(t)$ are realvalued functions defined on $\square$ such that $r(t)>0$.

By a solution of (9) on the interval $\mathbb{\complement} \subseteq$, we mean a nontrivial real-valued function $x \in C^{2}(\mathbb{J})$, which has the

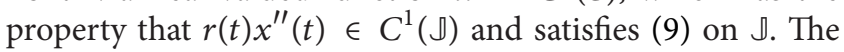
nontrivial solution $x(t)$ of (9) is said to oscillate or to be oscillatory if it has arbitrarily large zeros. Equation (9) is oscillatory if one of its nontrivial solutions is oscillatory. An equation of the form (9) is said to be disconjugate on an interval $\square$ if no nontrivial solution has more than two zeros

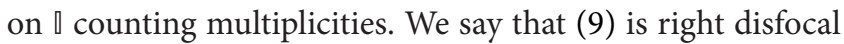
(left disfocal) on the interval $[a, b],(a<b)$ if the solutions of (9) such that $x^{\prime}(a)=0, x(a) \neq 0\left(x^{\prime}(b)=0\right.$, and $\left.x(b) \neq 0\right)$ do not have two zeros counting multiplicities in $(a, b]([a, b))$.

The paper is organized as follows. In Section 2, we present Hardy's inequality, the extensions of Opial's inequality, and Wirtinger's inequality that will be used to prove our main results. In Section 3, we are concerned with the lower bounds of the distance between zeros of a nontrivial solution and/or its derivatives for the third-order differential equation (9) subject to two sets of boundary conditions. In particular, we will prove the following.

(i) Obtain lower bounds for the spacing $\beta-\alpha$, where $x$ is a nontrivial solution of (9) which satisfies

$$
\begin{aligned}
& x(\alpha)=x^{\prime}(\alpha)=x^{\prime}(\beta)=0, \quad \text { or } \\
& x(\beta)=x^{\prime}(\beta)=x^{\prime}(\alpha)=0 .
\end{aligned}
$$

(ii) Obtain lower bounds for the spacing $\beta-\alpha$, where $x$ is a solution of (9) which satisfies

$$
\begin{aligned}
& x(\alpha)=x^{\prime}(\alpha)=x^{\prime \prime}(\beta)=0, \quad \text { or } \\
& x(\beta)=x^{\prime}(\beta)=x^{\prime \prime}(\alpha)=0 .
\end{aligned}
$$

\section{Hardy, Opial, and Wirtinger Inequalities}

In this section, we present Hardy's inequality and some generalizations of Opial and Wirtinger type inequalities that will be needed in the proof of the main results. The generalizations of Opial and Wirtinger type inequalities are adapted from Agarwal and Pang $[18,19]$ and the papers due to Beesack and Das [20], Clark and Hinton [21], and Fink [22]. The Hardy inequality is adapted from the book due to Kufner and Persson [23]. We begin with Hardy's inequality which states the following. If $y$ is absolutely continuous on $(\alpha, \beta)$, such that $y(\alpha)=0$ or $(y(\beta)=0)$, then

$$
\left(\int_{\alpha}^{\beta} p(t)|y(t)|^{n} d t\right)^{1 / n} \leq C\left(\int_{\alpha}^{\beta} r(t)\left|y^{\prime}(t)\right|^{m} d t\right)^{1 / m},
$$


Mathematical Problems in Engineering

3

where the weighted functions $p, r$ are positive functions defined on $(\alpha, \beta)$ and $n, m$ are real parameters that satisfy $0<n \leq \infty$ and $1 \leq m \leq \infty$. The constant $C$ is given by

$$
C \leq m^{1 / m}\left(m_{1}\right)^{1 / m_{1}} A(\alpha, \beta), \quad \text { for } 1<m \leq n, m_{1}=\frac{m}{m-1}
$$

where

$$
\begin{array}{r}
A(\alpha, \beta):=\sup _{\alpha \leq t \leq \beta}\left(\int_{t}^{\beta} p(t) d t\right)^{1 / n}\left(\int_{\alpha}^{t} r^{1-m_{1}}(s) d s\right)^{1 / m_{1}}, \\
\text { if } y(\alpha)=0, \\
A(\alpha, \beta):=\sup _{\alpha \leq t \leq \beta}\left(\int_{\alpha}^{t} p(t) d t\right)^{1 / n}\left(\int_{t}^{\beta} r^{1-m_{1}}(s) d s\right)^{1 / m_{1}}, \\
\text { if } y(\beta)=0 .
\end{array}
$$

Note that inequality (12) has an immediate application to the case when $y(\alpha)=y(\beta)=0$. In this case, inequality (12) is satisfied if and only if

$$
\begin{aligned}
& A(\alpha, \beta) \\
& :=\sup _{(c, d) \subset(\alpha, \beta)}\left(\int_{c}^{d} p(t) d t\right)^{1 / n} \\
& \quad \times \min \left\{\left(\int_{\alpha}^{c} r^{1-m_{1}}(s) d s\right)^{1 / n_{1}},\left(\int_{d}^{\beta} r^{1-m_{1}}(s) d s\right)^{1 / m_{1}}\right\}
\end{aligned}
$$

exists and is finite. The Opal type inequality due to Beesack which is a generalization of Opal's inequality states that if $y$ is absolutely continuous on $[\alpha, \beta]$ with $y(\alpha)=0$, then the following inequality holds:

$$
\int_{\alpha}^{\beta} q(t)|y(t)|^{m}\left|y^{\prime}(t)\right|^{n} d t \leq K_{1}(m, n) \int_{\alpha}^{\beta} p(t)\left|y^{\prime}(t)\right|^{m+n} d t,
$$

where $m, n$ are real numbers such that $m n>0, m+n>1$, $q(t)$, and $p(t)$ are nonnegative, measurable functions defined on $(\alpha, \beta)$ such that $\int_{\alpha}^{t}(p(s))^{-1 /(m+n-1)} d s<\infty$, and

$$
\begin{aligned}
& K_{1}(m, n) \\
& :=\left(\frac{n}{m+n}\right)^{n /(m+n)} \\
& \quad \times\left[\int_{\alpha}^{\beta} q^{(m+n) / n}(t) p^{-n / m}(t)\right. \\
& \left.\quad \times\left(\int_{\alpha}^{t}(p(s))^{-1 /(m+n-1)} d s\right)^{m+n-1} d t\right]^{m /(m+n)} .
\end{aligned}
$$

If instead $y(\beta)=0$, then (16) holds, where $K_{1}(m, n)$ is replaced by

$$
\begin{aligned}
& K_{2}(m, n) \\
& :=\left(\frac{n}{m+n}\right)^{n /(m+n)} \\
& \quad \times\left[\int_{\alpha}^{\beta} q^{(m+n) / n}(t) p^{-(n / m)}(t)\right. \\
& \left.\quad \times\left(\int_{t}^{\beta}(p(s))^{-1 /(m+n-1)} d s\right)^{m+n-1} d t\right]^{m /(m+n)} .
\end{aligned}
$$

The Opal type inequality due to Agarwal and Pang states that if $y(t) \in C^{(n-1)}[\alpha, \beta]$ and satisfies $y^{(i)}(\alpha)=0,0 \leq k \leq i \leq n-1$ $(n \geq 1)$ and $y^{(n-1)}(t)$ absolutely continuous on $(\alpha, \beta)$, then

$$
\begin{aligned}
& \int_{\alpha}^{\beta} \phi(t)\left|y^{(k)}(t)\right|^{l}\left|y^{(n)}(t)\right|^{m} d t \\
& \quad \leq H_{1}\left[\int_{\alpha}^{\beta} \varphi(t)\left|y^{(n)}(t)\right|^{c} d t\right]^{(l+m) / c},
\end{aligned}
$$

where $\phi$ and $\varphi$ are nonnegative and measurable function defined on $(\alpha, \beta), m, n$ are real numbers such that $c / m>1$, and

$$
\begin{aligned}
& H_{1}:= \frac{(m /(m+l))^{m / c}}{(n-k-1) !} \\
& \times\left[\int_{\alpha}^{\beta}\left(\phi^{c}(t) \varphi^{-m}(t)\right)^{1 /(c-m)}\right. \\
&\left.\quad \times\left(G_{1, k}(t)\right)^{l(c-1) /(c-m)} d t\right]^{(c-m) / c}, \\
& G_{1, k}(t):=\int_{\alpha}^{t}(t-s)^{(n-k-1) c /(c-1)}(\varphi(s))^{-1 /(c-1)} d s .
\end{aligned}
$$

If instead $y^{(i)}(\beta)=0,0 \leq k \leq i \leq n-1(n \geq 1)$, then (19) holds, where $H_{1}$ is replaced by

$$
\begin{aligned}
& H_{2}:= \frac{(m /(m+l))^{m / c}}{(n-k-1) !} \\
& \times\left[\int_{\alpha}^{\beta}\left(\phi^{c}(t) \varphi^{-m}(t)\right)^{1 /(c-m)}\right. \\
&\left.\quad \times\left(G_{2, k}(t)\right)^{l(c-1) /(c-m)} d t\right]^{(c-m) / c}, \\
& G_{2, k}(t):=\int_{t}^{\beta}(s-t)^{(n-k-1) c /(c-1)}(\varphi(s))^{-1 /(c-1)} d s .
\end{aligned}
$$


We also need the following inequality, which is the special case of an inequality proved by Agarwal and Pang [18] with two functions:

$$
\int_{\alpha}^{\beta} c(t)\left|y^{(k)}(t)\right|\left|y^{(k+1)}(t)\right| d t \leq C_{\alpha} \int_{\alpha}^{\beta} r(t)\left|y^{(n)}(t)\right|^{2} d t
$$

where $y(t) \in C^{n-1}[\alpha, \beta]$ and satisfies $y^{(i)}(\alpha)=0, k \leq i \leq n-1$, $0 \leq k \leq n-1(n \geq 1)$, and $y^{(n-1)}$ is absolutely continuous on $(\alpha, \beta), c(t)$, and $r(t)$, being nonnegative measurable functions defined on $(\alpha, \beta)$, and

$$
C_{\alpha}:=\frac{1}{2((n-k-1) !)^{2}} \max _{t \in[\alpha, \beta]} c(t) \int_{\alpha}^{\beta} \frac{(s-\alpha)^{2(n-k-1)}}{r(s)} d s .
$$

If $y^{(i)}(\beta)=0, k \leq i \leq n-1$, then (22) holds, where $C_{\alpha}$ is replaced:

$$
C_{\beta}:=\frac{1}{2((n-k-1) !)^{2}} \max _{t \in[\alpha, \beta]} c(t) \int_{\alpha}^{\beta} \frac{(\beta-s)^{2(n-k-1)}}{r(s)} d s .
$$

The Wirtinger type inequality due to Agarwal and Pang states that if $y(\alpha)=y(\beta)=0$, then

$$
\begin{aligned}
\int_{\alpha}^{\beta} y^{\gamma+1}(t) d t \leq & \frac{(\beta-\alpha)^{\gamma+1} \Gamma^{2}((\gamma+2) / 2)}{2 \Gamma(\gamma+2)} \\
& \times \int_{\alpha}^{\beta}\left(y^{\prime}(t)\right)^{\gamma+1}(t) d t .
\end{aligned}
$$

The Opial type inequality due to Clark and Hinton inequality states that if $y \in C^{2}[\alpha, \beta]$, where $y(\alpha)=y^{\prime}(\alpha)=0$, then

$$
\left(\int_{\alpha}^{\beta}|y(t)|^{2}\left|y^{\prime \prime}(t)\right|^{2} d t\right)^{1 / r} \leq \frac{(\beta-\alpha)^{3 / 2}}{\sqrt{3}} \int_{\alpha}^{\beta}\left|y^{\prime \prime}(t)\right|^{2} d t .
$$

The Opial type inequality due to Fink inequality states that if $y^{(i)}(\alpha)=0,0 \leq i \leq n-1, \mu \geq 1,(1 / \mu)+(1 / \nu)=1$, then

$$
\begin{aligned}
& \int_{\alpha}^{\beta}\left|y^{(k)}(t) y^{(r)}(t)\right| d t \\
& \quad \leq C(n, k, r, \mu)(\beta-\alpha)^{2 n-k-r+1-2 / \mu}\left(\int_{\alpha}^{\beta}\left|y^{(n)}(t)\right|^{\mu} d t\right)^{2 / \mu},
\end{aligned}
$$

where $0 \leq k<r<n,(n \geq 2)$, and

$$
C(n, k, r, \mu)=\frac{1}{2((n-k-1) !)^{2}[(n-k-1) \nu+1]^{2 / \nu}}
$$

\section{Main Results}

In this section, we state and prove the main results. For simplicity, we introduce the following notations:

$$
\begin{gathered}
H_{1}\left(\alpha, \beta, G_{1,0}\right)=\frac{1}{\sqrt{2}}\left[\int_{\alpha}^{\beta} Q_{1}^{2}(t) \frac{G_{1}(t)}{r(t)} d t\right]^{1 / 2}, \\
G_{1}(t)=\int_{\alpha}^{t} \frac{(t-s)^{2}}{r(s)} d s, \quad Q_{1}(t)=\int_{t}^{\beta} q(s) d s \\
H_{2}\left(\alpha, \beta, G_{2}\right)=\frac{1}{\sqrt{2}}\left[\int_{\alpha}^{\beta} Q_{2}^{2}(t) \frac{G_{2}(t)}{r(t)} d t\right]^{1 / 2}, \\
G_{2}(t)=\int_{t}^{\beta} \frac{(t-s)^{2}}{r(s)} d s, \quad Q_{2}(t)=\int_{\alpha}^{t} q(s) d s, \\
A_{i}(\alpha, \beta)=J_{i}(t) \min \left\{\left(\int_{\alpha}^{c} \frac{1}{r(s)} d s\right)^{1 / 2},\left(\int_{c}^{\beta} \frac{1}{r(s)} d s\right)^{1 / 2}\right\}
\end{gathered}
$$

where $J_{i}(t)=\sup _{(c, d) \subset(\alpha, \beta)}\left(\int_{\alpha}^{\beta} D_{i}(t) d t\right)^{1 / 2}$ and $\left|D_{i}(t)\right|=$ $\left|Q_{i}(t)\right|+|p(t)|, i=1,2$,

$$
\begin{aligned}
& B_{1}(\alpha, \beta):=\sup _{\alpha \leq t \leq \beta}\left(\int_{t}^{\beta} p(t) d t\right)^{1 / 2}\left(\int_{\alpha}^{t} \frac{1}{r(s)} d s\right)^{1 / 2}, \\
& C_{\alpha}:=\frac{1}{2} \max _{\alpha \leq t \leq \beta} q(t) \int_{\alpha}^{\beta} \frac{(s-\alpha)^{2}}{r(s)} d s, \\
& B_{2}(\alpha, \beta):=\sup _{\alpha \leq t \leq \beta}\left(\int_{\alpha}^{t} p(t) d t\right)^{1 / 2}\left(\int_{t}^{\beta} \frac{1}{r(s)} d s\right)^{1 / 2}, \\
& C_{\beta}:=\frac{1}{2} \max _{\alpha \leq t \leq \beta} q(t) \int_{\alpha}^{\beta} \frac{(\beta-s)^{2}}{r(s)} d s, \\
& \psi(\alpha, \beta):=\sup _{\alpha \leq t \leq \beta}\left(\int_{t}^{\beta} r(t) d t\right)^{1 / 2}\left(\int_{\alpha}^{t} \frac{1}{r(s)} d s\right)^{1 / 2} .
\end{aligned}
$$

Now, we are ready to state and prove the main results.

Theorem 1. Assume that $x(t)$ is a nontrivial solution of (9). If $x(\alpha)=x^{\prime}(\alpha)=x^{\prime}(\beta)=0$, then

$$
H_{1}\left(\alpha, \beta, G_{1}\right)+4 A_{1}^{2}(\alpha, \beta) \geq 1 \text {. }
$$

If $x^{\prime}(\alpha)=x(\beta)=x^{\prime}(\beta)=0$, then

$$
H_{2}\left(\alpha, \beta, G_{2}\right)+4 A_{2}^{2}(\alpha, \beta) \geq 1
$$

Proof. We prove (35). Multiplying (9) by $x^{\prime}(t)$ and integrating the new equation from $\alpha$ to $\beta$, we obtain

$$
\int_{\alpha}^{\beta} x^{\prime}\left(r\left(x^{\prime \prime}\right)\right)^{\prime} d t=-\int_{\alpha}^{\beta} p\left(x^{\prime}\right)^{2} d t-\int_{\alpha}^{\beta} q x x^{\prime} d t .
$$


Integrating by parts the left-hand side, we get that

$$
\left.r x^{\prime} x^{\prime \prime}\right|_{\alpha} ^{\beta}-\int_{\alpha}^{\beta} r\left(x^{\prime \prime}\right)^{2} d t=-\int_{\alpha}^{\beta} p\left(x^{\prime}\right)^{2} d t-\int_{\alpha}^{\beta} q x x^{\prime} d t .
$$

Using the assumptions that $x^{\prime}(\alpha)=x^{\prime}(\beta)=0$ and $Q_{1}(t)=$ $\int_{t}^{\beta} q(s) d s$, we have

$$
\begin{aligned}
\int_{\alpha}^{\beta} r(t)\left(x^{\prime \prime}(t)\right)^{2} d t= & \int_{\alpha}^{\beta} p(t)\left(x^{\prime}(t)\right)^{2} d t \\
& +\int_{\alpha}^{\beta} Q_{1}^{\prime}(t) x(t) x^{\prime}(t) d t .
\end{aligned}
$$

Integrating the term $\int_{\alpha}^{\beta} Q_{1}^{\prime}(t) x(t) x^{\prime}(t) d s$ by parts and using the assumption that $x^{\prime}(\alpha)=x^{\prime}(\beta)=0$, we obtain

$$
\begin{aligned}
& \int_{\alpha}^{\beta} Q_{1}^{\prime}(t) x(t) x^{\prime}(t) d s \\
& \quad=-\int_{\alpha}^{\beta} Q_{1}(t)\left(x^{\prime}(t)\right)^{2} d t-\int_{\alpha}^{\beta} Q_{1}(t) x(t) x^{\prime \prime}(t) d t .
\end{aligned}
$$

Substituting (40) into (39), we get

$$
\begin{aligned}
\int_{\alpha}^{\beta} r(t)\left|x^{\prime \prime}(t)\right|^{2} d t \leq & \int_{\alpha}^{\beta}\left|D_{1}(t)\right|\left|x^{\prime}(t)\right|^{2} d t \\
& +\int_{\alpha}^{\beta}\left|Q_{1}(t)\right||x(t)|\left|x^{\prime \prime}(t)\right| d t,
\end{aligned}
$$

where $\left|D_{1}(t)\right|=\left|Q_{1}(t)\right|+|p(t)|$. Applying inequality (19) on the integral

$$
\int_{\alpha}^{\beta}\left|Q_{1}(t)\right||x(t)|\left|x^{\prime \prime}(t)\right| d t
$$

with $\phi(t)=\left|Q_{1}(t)\right|, \varphi(t)=r(t), k=0, m=l=1, n=2, c=2$, and $x(\alpha)=x^{\prime}(\alpha)=0$, we get

$$
\begin{aligned}
& \int_{\alpha}^{\beta}\left|Q_{1}(t)\right||x(t)|\left|x^{\prime \prime}(t)\right| d t \\
& \quad \leq H_{1}\left(\alpha, \beta, G_{1}\right) \int_{\alpha}^{\beta} r(t)\left|x^{\prime \prime}(t)\right|^{2} d t,
\end{aligned}
$$

where $H_{1}\left(\beta, \alpha, G_{1}\right)$ is defined as in (29). Applying inequality (12) on the integral $\int_{\alpha}^{\beta}\left|D_{1}(t)\right|\left|x^{\prime}(t)\right|^{2} d t$ with $y(t)=x^{\prime}(t)$ and $y(\alpha)=y(\beta)=0$, we have that

$$
\int_{\alpha}^{\beta}\left|D_{1}(t)\right|\left|x^{\prime}(t)\right|^{2} d t \leq 4 A_{1}^{2}(\alpha, \beta) \int_{\alpha}^{\beta} r(t)\left|x^{\prime \prime}(t)\right|^{2} d t
$$

where $A_{1}(\alpha, \beta)$ is defined as in (31). Substituting (44) and (43) into (41), we get

$$
\begin{aligned}
\int_{\alpha}^{\beta}|r(t)|\left|x^{\prime \prime}(t)\right|^{2} d t \leq & 4 A_{1}^{2}(\alpha, \beta) \int_{\alpha}^{\beta} r(t)\left|x^{\prime \prime}(t)\right|^{2} d t \\
& +H_{1}\left(\alpha, \beta, G_{1}\right) \int_{\alpha}^{\beta} r(t)\left|x^{\prime \prime}(t)\right|^{2} d t .
\end{aligned}
$$

Cancelling the term $\int_{\alpha}^{\beta} r(t)\left|x^{\prime \prime}(t)\right|^{2} d t$, we get

$$
4 A_{1}^{2}(\alpha, \beta)+H_{1}\left(\alpha, \beta, G_{1}\right) \geq 1,
$$

which is the desired inequality (35). The proof of (36) is similar to the proof of (35) by replacing $H_{1}\left(\alpha, \beta, G_{1}\right)$ by $H_{2}\left(\alpha, \beta, G_{2}\right)$ and $A_{1}(\alpha, \beta)$ by $A_{2}(\alpha, \beta)$. The proof is complete.

In the following, we apply the Clark and Hinton inequality (26) to get a new result.

Theorem 2. Assume that $r(t)$ is a nonincreasing function and suppose that $x(t)$ is a solution of (9). If $x(\alpha)=x^{\prime}(\alpha)=x^{\prime}(\beta)=$ 0 , then

$$
4 A_{1}^{2}(\alpha, \beta)+\frac{(\beta-\alpha)^{3 / 2}}{\sqrt{3} r(\beta)}\left(\int_{\alpha}^{\beta}\left|Q_{1}(t)\right|^{2} d t\right)^{1 / 2} \geq 1,
$$

where $A_{1}(\alpha, \beta)$ is defined as in (31) for $i=1$ and $Q_{1}(t)$ is defined as in (29).

Proof. Multiply (9) by $x^{\prime}(t)$ and proceed as in the proof of Theorem 1 to get

$$
\begin{aligned}
\int_{\alpha}^{\beta} r(t)\left|x^{\prime \prime}(t)\right|^{2} d t \leq & \int_{\alpha}^{\beta}\left|D_{1}(t)\right|\left|x^{\prime}(t)\right|^{2} d t \\
& +\int_{\alpha}^{\beta}\left|Q_{1}(t)\right||x(t)|\left|x^{\prime \prime}(t)\right| d t,
\end{aligned}
$$

where $\left|D_{1}(t)\right|=\left|Q_{1}(t)\right|+|p(t)|$. Applying the Schwarz inequality

$$
\int_{\alpha}^{\beta}|f(t) g(t)| d t \leq\left(\int_{\alpha}^{\beta}|f(t)|^{2} d t\right)^{1 / 2}\left(\int_{\alpha}^{\beta}|g(t)|^{2} d t\right)^{1 / 2},
$$

on the integral $\int_{\alpha}^{\beta}\left|Q_{1}(t)\|x(t)\| x^{\prime \prime}(t)\right| d t$, we have that

$$
\begin{aligned}
\int_{\alpha}^{\beta}\left|Q_{1}(t)\right||x(t)|\left|x^{\prime \prime}(t)\right| d t \leq & \left(\int_{\alpha}^{\beta}\left|Q_{1}(t)\right|^{2} d t\right)^{1 / 2} \\
& \times\left(\int_{\alpha}^{\beta}|x(t)|^{2}\left|x^{\prime \prime}(t)\right|^{2} d t\right)^{1 / 2} .
\end{aligned}
$$

Applying inequality (26) and using the assumption $x(\alpha)=$ $x^{\prime}(\alpha)=0$, we get that

$$
\left(\int_{\alpha}^{\beta}|x(t)|^{2}\left|x^{\prime \prime}(t)\right|^{2} d t\right)^{1 / 2} \leq \frac{(\beta-\alpha)^{3 / 2}}{\sqrt{3}} \int_{\alpha}^{\beta}\left|x^{\prime \prime}(t)\right|^{2} d t
$$

Substituting (51) into (50) and using the assumption that $r(t)$ is a nonincreasing function, we have

$$
\begin{aligned}
& \int_{\alpha}^{\beta}\left|Q_{1}(t)\right||x(t)|\left|x^{\prime \prime}(t)\right| \\
& \quad \leq \frac{(\beta-\alpha)^{3 / 2}}{\sqrt{3} r(\beta)}\left(\int_{\alpha}^{\beta}\left|Q_{1}(t)\right|^{2} d t\right)^{1 / 2} \int_{\alpha}^{\beta} r(t)\left|x^{\prime \prime}(t)\right|^{2} d t .
\end{aligned}
$$


Applying Hardy's inequality (12) on the integral $\int_{\alpha}^{\beta}\left|D_{1}(t)\right|\left|x^{\prime}(t)^{2}\right| d t$, we obtain

$$
\int_{\alpha}^{\beta}\left|D_{1}(t)\right|\left|x^{\prime}(t)\right|^{2} d t \leq 4 A_{1}^{2}(\alpha, \beta) \int_{\alpha}^{\beta} r(t)\left|x^{\prime \prime}(t)\right|^{2} d t,
$$

where $x^{\prime}(\alpha)=x^{\prime}(\beta)=0$. Substituting (52) and (53) into (48), we obtain

$$
\begin{aligned}
& \int_{\alpha}^{\beta}|r(t)|\left|x^{\prime \prime}(t)\right|^{2} d t \\
& \leq 4 A_{1}^{2}(\alpha, \beta) \int_{\alpha}^{\beta} r(t)\left|x^{\prime \prime}(t)\right|^{2} d t \\
& \quad+\frac{(\beta-\alpha)^{3 / 2}}{\sqrt{3} r(\beta)}\left(\int_{\alpha}^{\beta}\left|Q_{1}(t)\right|^{2} d t\right)^{1 / 2} \int_{\alpha}^{\beta} r(t)\left|x^{\prime \prime}(t)\right|^{2} d t .
\end{aligned}
$$

The desired inequality (47) followed by cancelling the term $\int_{\alpha}^{\beta}|r(t)|\left|x^{\prime \prime}(t)\right|^{2} d t$. The proof is complete.

The proof of the following theorem is similar to the proof of Theorem 2.

Theorem 3. Assume that $r(t)$ is a nonincreasing function and suppose that $x(t)$ is a solution of $(9)$. If $x^{\prime}(\alpha)=x(\beta)=x^{\prime}(\beta)=$ 0 , then

$$
4 A_{2}^{2}(\alpha, \beta)+\frac{(\beta-\alpha)^{3 / 2}}{\sqrt{3} r(\beta)}\left(\int_{\alpha}^{\beta}\left|Q_{1}(t)\right|^{2} d t\right)^{1 / 2} \geq 1,
$$

where $A_{2}(\alpha, \beta)$ is defined as in (31) for $i=2$ and $Q_{2}(t)$ is defined as in (30).

One can use Theorems 2 and 3 to obtain some different special cases. For example, as a special case of Theorem 2, when $r(t)=1$ and $p(t)=0$, we have the following result.

Corollary 4. If $x(t)$ is a nontrivial solution of

$$
x^{\prime \prime \prime}(t)+q(t) x(t)=0, \quad t \in[\alpha, \beta],
$$

which satisfies $x(\alpha)=x^{\prime}(\alpha)=x^{\prime}(\beta)=0$, then

$$
\int_{\alpha}^{\beta}\left|Q_{1}(t)\right|^{2} d t \geq \frac{3}{(\beta-\alpha)^{3}}
$$

where $Q_{1}(t)$ is defined as in (29).

Now, we will prove a new result when $r(t)=1$.

Theorem 5. Suppose that $r(t)=1$ and assume that $x(t)$ is a solution of $(9)$. If $x(\alpha)=x^{\prime}(\alpha)=x^{\prime}(\beta)=0$, or $x(\beta)=x^{\prime}(\beta)=$ $x^{\prime}(\alpha)=0$, then

$$
P \frac{\pi(\beta-\alpha)^{2}}{16}+Q \frac{(\beta-\alpha)^{3}}{6} \geq 1,
$$

where $P=\max _{\alpha \leq t \leq \beta}|p(t)|$ and $Q=\max _{\alpha \leq t \leq \beta}|q(t)|$.
Proof. Multiplying (9) by $x^{\prime}$ and integrating by parts the lefthand side, we have

$$
\begin{aligned}
& \left.x^{\prime}(t) x^{\prime \prime}(t)\right|_{\alpha} ^{\beta}-\int_{\alpha}^{\beta}\left(x^{\prime \prime}(t)\right)^{2} d t \\
& =-\int_{\alpha}^{\beta} p(t)\left(x^{\prime}(t)\right)^{2} d t-\int_{\alpha}^{\beta} q(t) x(t) x^{\prime}(t) d t .
\end{aligned}
$$

Using the assumptions that $x^{\prime}(\alpha)=x^{\prime}(\beta)=0$, we obtain

$$
\int_{\alpha}^{\beta}\left(x^{\prime \prime}\right)^{2} d t=\int_{\alpha}^{\beta} p\left(x^{\prime}\right)^{2} d t+\int_{\alpha}^{\beta} q x x^{\prime} d t .
$$

By using the maximum values of $|p(t)|$ and $|q(t)|$, we can write (60) as

$$
\int_{\alpha}^{\beta}\left|x^{\prime \prime}(t)\right|^{2} d t \leq P \int_{\alpha}^{\beta}\left|x^{\prime}(t)\right|^{2} d t+Q \int_{\alpha}^{\beta}|x(t)|\left|x^{\prime}(t)\right| d t .
$$

For the first term on the right-hand side $\int_{\alpha}^{\beta}\left|x^{\prime}(t)\right|^{2} d t$, we apply inequality (25) with $y(t)=x^{\prime}(t)$ (note that $x^{\prime}(\alpha)=$ $\left.x^{\prime}(\beta)=0\right)$ and $\gamma=1$, to obtain

$$
\int_{\alpha}^{\beta}\left|x^{\prime}\right|^{2} d t \leq \frac{\pi(\beta-\alpha)^{2}}{16} \int_{\alpha}^{\beta}\left|x^{\prime \prime}\right|^{2} d t
$$

Applying Fink inequality (27) on $\int_{\alpha}^{\beta}|x|\left|x^{\prime}\right| d t$, with $k=0, r=$ $1, \mu=\nu=2$, and $n=2$, we have

$$
\int_{\alpha}^{\beta}|x|\left|x^{\prime}\right| d t \leq \frac{(\beta-\alpha)^{3}}{6} \int_{\alpha}^{\beta}\left|x^{\prime \prime}(t)\right|^{2} d t .
$$

Substituting (62) and (63) into (61), we get, after cancelling the term $\int_{\alpha}^{\beta}\left|x^{\prime \prime}(t)\right|^{2} d t$, that

$$
P \frac{\pi(\beta-\alpha)^{2}}{16}+Q \frac{(\beta-\alpha)^{3}}{6} \geq 1,
$$

which is the desired inequality (58). The proof is complete.

As a special case of Theorem 5 , when $p(t)=0$, we have the following result.

Corollary 6. Let $x(t)$ be a nontrivial solution of (56). If $x(\alpha)=$ $x^{\prime}(\alpha)=x^{\prime}(\beta)=0$, then

$$
\max _{\alpha \leq t \leq \beta}|q(t)| \geq \frac{6}{(\beta-\alpha)^{3}} .
$$

In the following, we prove some results related to the boundary conditions presented in (ii).

Theorem 7. Assume that $x(t)$ is a nontrivial solution of (9). If $x(\alpha)=x^{\prime}(\alpha)=x^{\prime \prime}(\beta)=0$, then

$$
4 B_{1}^{2}(\alpha, \beta)+C_{\alpha} \geq 1 .
$$

If $x(\beta)=x^{\prime}(\beta)=x^{\prime \prime}(\alpha)=0$, then

$$
4 B_{2}^{2}(\alpha, \beta)+C_{\beta} \geq 1 \text {. }
$$


Proof. Multiplying (9) by $x^{\prime}(t)$ and integrating by parts the left-hand side from $\alpha$ to $\beta$, we get

$$
\left.r x^{\prime} x^{\prime \prime}\right|_{\alpha} ^{\beta}-\int_{\alpha}^{\beta} r\left(x^{\prime \prime}\right)^{2} d t=-\int_{\alpha}^{\beta} p\left(x^{\prime}\right)^{2} d t-\int_{\alpha}^{\beta} q x x^{\prime} d t
$$

Using the assumption that $x^{\prime}(\alpha)=x^{\prime \prime}(\beta)=0$, we have

$$
\begin{aligned}
\int_{\alpha}^{\beta} r(t)\left|x^{\prime \prime}(t)\right|^{2} d t \leq & \int_{\alpha}^{\beta}|p(t)|\left|x^{\prime}(t)\right|^{2} d t \\
& +\int_{\alpha}^{\beta}|q(t)||x(t)|\left|x^{\prime}(t)\right| d t .
\end{aligned}
$$

Applying inequality (12) on the integral $\int_{\alpha}^{\beta}|p(t)|\left|x^{\prime}(t)\right|^{2} d t$ with $n=m=2$, we get

$$
\int_{\alpha}^{\beta}|p(t)|\left|x^{\prime}(t)\right|^{2} d t \leq 4 B_{1}^{2}(\alpha, \beta) \int_{\alpha}^{\beta}|r(t)|\left|x^{\prime \prime}(t)^{2}\right| d t
$$

where $B_{1}(\alpha, \beta)$ is defined as in (32). Again applying inequality (22) on the integral $\int_{\alpha}^{\beta}|q(t) \| x(t)|\left|x^{\prime}(t)\right| d t$, we get

$$
\int_{\alpha}^{\beta}|q(t)||x(t)|\left|x^{\prime}(t)\right| d t \leq C_{\alpha} \int_{\alpha}^{\beta}|r(t)|\left|x^{\prime \prime}(t)^{2}\right| d t,
$$

where $C_{\alpha}$ is defined as in (32). Substituting (70) and (71) into (69), we obtain

$$
\begin{aligned}
& \int_{\alpha}^{\beta}|r(t)|\left|x^{\prime \prime}(t)\right|^{2} d t \\
& \quad \leq 4 B_{1}^{2} \int_{\alpha}^{\beta}|r(t)|\left|x^{\prime \prime}(t)^{2}\right| d t+C_{\alpha} \int_{\alpha}^{\beta}|r(t)|\left|x^{\prime \prime}(t)^{2}\right| d t .
\end{aligned}
$$

By cancelling the term $\int_{\alpha}^{\beta}|r(t)|\left|x^{\prime \prime}(t)^{2}\right| d t$, we get that

$$
4 B_{1}^{2}(\alpha, \beta)+C_{\alpha} \geq 1
$$

which is the desired result (66). The proof (67) is similar to the proof of (66) by using $B_{2}(\alpha, \beta)$ and $C_{\beta}$ instead of $B_{1}(\alpha, \beta)$ and $C_{\alpha}$ and hence is omitted. The proof is complete.

In the following, we apply an inequality due to Boyd [24] to obtain new results. The Boyd inequality states that if $y \in$ $C^{1}[\alpha, \beta]$, with $y(\alpha)=0($ or $y(\beta)=0)$, then

$$
\begin{aligned}
& \int_{\alpha}^{\beta}|y(t)|^{\nu}\left|y^{\prime}(t)\right|^{\eta} d t \\
& \quad \leq N(\nu, \eta, s)(\beta-\alpha)^{\nu}\left[\int_{a}^{b}\left|y^{\prime}(t)\right|^{s} d t\right]^{(\nu+\eta) / s},
\end{aligned}
$$

where $v>0, s>1,0 \leq \eta<s$, and

$$
\begin{gathered}
N(\nu, \eta, s):=\frac{(s-\eta) \nu^{\nu} r^{\nu+\eta-s}}{(s-1)(\nu+\eta)(I(\nu, \eta, s))^{\nu}} \\
r:=\left\{\frac{\nu(s-1)+(s-\eta)}{(s-1)(\nu+\eta)}\right\}^{1 / s}, \\
I(\nu, \eta, s):=\int_{0}^{1}\left\{1+\frac{s(\eta-1)}{s-\eta} t\right\}^{-(\nu+\eta+s v) / s \nu} \\
\quad \times[1+(\eta-1)] t^{1 /(\nu-1)} d t .
\end{gathered}
$$

Note that an inequality of type (34) also holds when $y(\alpha)=$ $y(\beta)=0$. Choose $c=(\alpha+\beta) / 2$ and apply (34) to $[\alpha, c]$ and $[c, \beta]$ and by addition we obtain

$$
\begin{aligned}
& \int_{\alpha}^{\beta}|y(t)|^{\nu}\left|y^{\prime}(t)\right|^{\eta} d t \\
& \quad \leq N(\nu, \eta, s)\left(\frac{\beta-\alpha}{2}\right)^{\nu}\left[\int_{\alpha}^{\beta}\left|y^{\prime}(t)\right|^{s} d t\right]^{(\nu+\eta) / s},
\end{aligned}
$$

where $N(\nu, \eta, s)$ is defined as in (75). An inequality of type (74) holds when $\eta=s$ and $y(\alpha)=0$ (or $y(\beta)=0$ ). In this case, (74) becomes

$$
\begin{aligned}
& \int_{\alpha}^{\beta}|y(t)|^{\nu}\left|y^{\prime}(t)\right|^{\eta} d t \\
& \quad \leq L(\nu, \eta)(\beta-\alpha)^{\nu}\left[\int_{\alpha}^{\beta}\left|y^{\prime}(t)\right|^{\eta} d t\right]^{(\nu+\eta) / \eta},
\end{aligned}
$$

where

$$
L(\nu, \eta):=\frac{\eta \nu^{\eta}}{\nu+\eta}\left(\frac{\nu}{\nu+\eta}\right)^{\nu / \eta}\left(\frac{\Gamma(((\eta+1) / \eta)+(1 / \nu))}{\Gamma((\eta+1) / \eta) \Gamma(1 / \nu)}\right)^{\nu}
$$

and $\Gamma$ is the gamma function.

Theorem 8. Assume that $r(t)$ is nonincreasing function and $x(t)$ is a nontrivial solution of (9). If $x(\alpha)=x^{\prime}(\alpha)=x^{\prime \prime}(\beta)=0$, then

$$
4 B_{1}^{2}(\alpha, \beta)+\frac{8(\beta-\alpha)}{\pi r(\beta)} \psi^{2}(\alpha, \beta)\left(\int_{\alpha}^{\beta}|q(t)|^{2} d t\right)^{1 / 2} \geq 1
$$

If $x(\beta)=x^{\prime}(\beta)=x^{\prime \prime}(\alpha)=0$, then

$$
4 B_{2}^{2}(\alpha, \beta)+\frac{8(\beta-\alpha)}{\pi r(\beta)} \psi^{2}(\alpha, \beta)\left(\int_{\alpha}^{\beta}|q(t)|^{2} d t\right)^{1 / 2} \geq 1 .
$$

Proof. Multiplying (9) by $x^{\prime}(t)$ and integrating by parts the left-hand side, we have that

$$
\left.r x^{\prime} x^{\prime \prime}\right|_{\alpha} ^{\beta}-\int_{\alpha}^{\beta} r\left(x^{\prime \prime}\right)^{2} d t=-\int_{\alpha}^{\beta} p\left(x^{\prime}\right)^{2} d t-\int_{\alpha}^{\beta} q x x^{\prime} d t .
$$


Using the assumption $x^{\prime}(\alpha)=x^{\prime \prime}(\beta)=0$, we get that

$$
\begin{aligned}
\int_{\alpha}^{\beta}|r(t)|\left|x^{\prime \prime}(t)\right|^{2} d t \leq & \int_{\alpha}^{\beta}|p(t)|\left|x^{\prime}(t)\right|^{2} d t \\
& +\int_{\alpha}^{\beta}|q(t)||x(t)|\left|x^{\prime}(t)\right| d t .
\end{aligned}
$$

Applying inequality (12) on the integral $\int_{\alpha}^{\beta}|p(t)|\left|x^{\prime}(t)\right|^{2} d t$ with $m=n=2$, we get

$$
\begin{aligned}
& \int_{\alpha}^{\beta}|p(t)|\left|x^{\prime}(t)\right|^{2} d t \\
& \quad \leq 4 \psi^{2}(\alpha, \beta) \int_{\alpha}^{\beta}|r(t)|\left|x^{\prime \prime}(t)^{2}\right| d t,
\end{aligned}
$$

where $\psi(\alpha, \beta)$ is defined as in (34). Applying Schwarz's inequality on the term $\int_{\alpha}^{\beta}\left|q(t)\|x(t)\| x^{\prime}(t)\right| d t$, we get that

$$
\begin{aligned}
& \int_{\alpha}^{\beta}|q(t)||x(t)|\left|x^{\prime}(t)\right| d t \\
& \quad \leq\left(\int_{\alpha}^{\beta}|q(t)|^{2} d t\right)^{1 / 2}\left(\int_{\alpha}^{\beta}|x|^{2}\left|x^{\prime}\right|^{2} d t\right)^{1 / 2} .
\end{aligned}
$$

Applying again inequality (78) on the integral $\int_{\alpha}^{\beta}|x|^{2}\left|x^{\prime}\right|^{2} d t$ with $v=\eta=2$ (note that $x(\alpha)=0$ ) we obtain

$$
\int_{\alpha}^{\beta}|x|^{2}\left|x^{\prime}\right|^{2} d t \leq \frac{4(\beta-\alpha)^{2}}{\pi^{2} r^{2}(\beta)}\left[\int_{\alpha}^{\beta} r(t)\left|x^{\prime}\right|^{2} d t\right]^{2},
$$

where $r(t)$ is a nonincreasing function. Substituting (86) into (85), we get

$$
\begin{aligned}
& \int_{\alpha}^{\beta}|q(t)||x(t)|\left|x^{\prime}(t)\right| d t \\
& \quad \leq\left(\int_{\alpha}^{\beta}|q(t)|^{2} d t\right)^{1 / 2} \frac{2(\beta-\alpha)}{\pi r(\beta)} \int_{\alpha}^{\beta} r(t)\left|x^{\prime}\right|^{2} d t .
\end{aligned}
$$

Applying inequality (12) on the integral $\int_{\alpha}^{\beta} r(t)\left|x^{\prime}\right|^{2} d t$ with $p(t)=q(t)=r(t)$ and $m=n=2$, we get

$$
\int_{\alpha}^{\beta}|r(t)|\left|x^{\prime}(t)\right|^{2} d t \leq 4 B_{1}^{2}(\alpha, \beta) \int_{\alpha}^{\beta}|r(t)|\left|x^{\prime \prime}(t)\right|^{2} d t .
$$

Substituting (88) into (87), we have

$$
\begin{aligned}
& \int_{\alpha}^{\beta}|q(t)||x(t)|\left|x^{\prime}(t)\right| d t \\
& \leq\left(\int_{\alpha}^{\beta}|q(t)|^{2} d t\right)^{1 / 2} \frac{8(\beta-\alpha)}{\pi r(\beta)} \psi^{2}(\alpha, \beta) \\
& \quad \times \int_{\alpha}^{\beta}|r(t)|\left|x^{\prime \prime}(t)\right|^{2} d t .
\end{aligned}
$$

Substituting (84) and (89) into (83), we obtain

$$
\begin{aligned}
& \int_{\alpha}^{\beta}|r(t)|\left|x^{\prime \prime}(t)\right|^{2} d t \\
& \leq 4 B_{1}^{2}(\alpha, \beta) \int_{\alpha}^{\beta}|r(t)|\left|x^{\prime \prime}(t)\right|^{2} d t \\
& \quad+\left(\int_{\alpha}^{\beta}|q(t)|^{2} d t\right)^{1 / 2} \frac{8(\beta-\alpha)}{\pi r(\beta)} \psi^{2}(\alpha, \beta) \\
& \quad \times \int_{\alpha}^{\beta}|r(t)|\left|x^{\prime \prime}(t)\right|^{2} d t .
\end{aligned}
$$

Cancelling the term $\int_{\alpha}^{\beta}|r(t)|\left|x^{\prime \prime}(t)\right|^{2} d t$, we obtain

$$
4 B_{1}^{2}(\alpha, \beta)+\frac{8(\beta-\alpha)}{\pi r(\beta)} \psi^{2}(\alpha, \beta)\left(\int_{\alpha}^{\beta}|q(t)|^{2} d t\right)^{1 / 2} \geq 1
$$

which is the desired inequality (80). The proof of (81) is similar to the proof of $(80)$ by using $B_{2}(\alpha, \beta)$ instead of $B_{1}(\alpha, \beta)$. The proof is complete.

\section{Conflict of Interests}

The authors declare that there is no conflict of interests regarding the publication of this paper.

\section{References}

[1] E. Picard, "Mémoire sur la théorie des équations aux dérivés partielleset la méthode desapproximations successives," Journal de Mathématiques Pures et Appliquées, vol. 6, pp. 145-210, 1890.

[2] E. Picard, "Sur l'application des méthodes d'approximations successives à l'étude de certaines équations différentielles ordinaires," Journal de Mathématiques Pures et Appliquées, vol. 9, pp. 217-271, 1893.

[3] O. Niccoletti, "Sulle consizioni iniziali che determinano gli integrale delle equazioni diff eren ziali ordinarie," Atti della Accademia delle Scienze di Torino, vol. 33, pp. 746-759, 1897.

[4] C. de la Vallée Poussin, "Sur l'équation différentielle linéaire du second ordre," Journal de Mathématiques Pures et Appliquées, vol. 8, pp. 125-144, 1929.

[5] A. M. Lyapunov, "Probleme général de la stabilité du mouvement," Annales de la Faculté des sciences de Toulouse, vol. 9, pp. 203-474, 1907.

[6] S. H. Saker, "Some new disconjugacy criteria for second order differential equations with a middle term," Bulletin Mathematique de la Societe des Sciences Mathematiques de Roumanie, vol. 57, no. 1, pp. 109-120, 2014.

[7] G. Casadei, "Sul teorema di unicità di De La Vallée Poussin per equazioni differenziali del terzo ordine," Rendiconti del Seminario Matematico della Università di Padova, vol. 41, pp. 300-315, 1968.

[8] A. Lasota, "Sur la distance entre les zéros de l'équation différentielle linéaire du troisiéme ordre," Annales Polonici Mathematici, vol. 13, pp. 129-132, 1963. 
[9] R. M. Mathsen, "A disconjugacy condition for $y^{\prime \prime \prime}+a_{2} y^{\prime \prime}+a_{1} y^{\prime}+$ $a_{0} y=0$," Proceedings of the American Mathematical Society, vol. 17, pp. 627-632, 1966.

[10] B. G. Pachpatte, "On the zeros of solutions of certain differential equations," Demonstratio Mathematica, vol. 25, no. 4, pp. 825$833,1992$.

[11] B. G. Pachpatte, "Lyapunov type integral inequalities for certain differential equations," Georgian Mathematical Journal, vol. 4, no. 2, pp. 139-148, 1997.

[12] S. Panigrahi, "Lyapunov-type integral inequalities for certain higher-order differential equations," Electronic Journal of Differential Equations, vol. 2009, no. 28, pp. 1-14, 2009.

[13] N. Parhi and S. Panigrahi, "On Liapunov-type inequality for third-order differential equations," Journal of Mathematical Analysis and Applications, vol. 233, no. 2, pp. 445-460, 1999.

[14] N. Parhi and S. Panigrahi, "Disfocality and Liapunov-type inequalities for third-order equations," Applied Mathematics Letters, vol. 16, no. 2, pp. 227-233, 2003.

[15] U. Richard, "Metodi diversi per ottenere disequaglianze alla de la Vallée Poussin nelle equazioni differenziali ordinarie del secondo e terzo ordine," Rendiconti del Seminario Matematico Università e Politecnico di Torino, vol. 27, pp. 35-68, 1968.

[16] X. Yang, "On Liapunov-type inequality for certain higher-order differential equations," Applied Mathematics and Computation, vol. 134, no. 2-3, pp. 307-317, 2003.

[17] R. P. Agarwal and P. R. Krishnamurthy, "On the uniqueness of solution of nonlinear boundary value problems," Journal of Mathematical Sciences, vol. 10, no. 1, pp. 17-31, 1976.

[18] R. P. Agarwal and P. Y. Pang, Opial Inequalities with Applications in Differential and Difference Equation, Kluwer Academic, Dordrecht, The Netherlands, 1995.

[19] R. P. Agarwal and P. Y. Pang, "Remarks on the generalizations of Opial's inequality," Journal of Mathematical Analysis and Applications, vol. 190, no. 2, pp. 559-577, 1995.

[20] P. R. Beesack and K. M. Das, "Extensions of Opial's inequality," Pacific Journal of Mathematics, vol. 26, pp. 215-232, 1968.

[21] S. Clark and D. Hinton, "Some disconjugacy criteria for differential equations with oscillatory coefficients," Mathematische Nachrichten, vol. 278, no. 12-13, pp. 1476-1489, 2005.

[22] A. M. Fink, "On Opials inequality for $f^{(n)}$," Proceedings of the American Mathematical Society, vol. 155, pp. 177-181, 1992.

[23] A. Kufner and L.-E. Persson, Weighted Inequalities of Hardy Type, World Scientific, River Edge, NJ, USA, 2003.

[24] D. W. Boyd, "Best constants in a class of integral inequalities," Pacific Journal of Mathematics, vol. 30, pp. 367-383, 1969. 


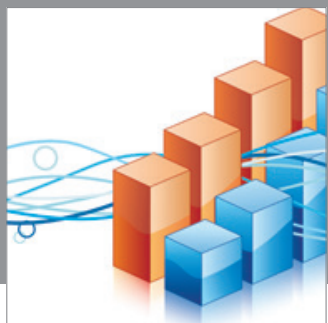

Advances in

Operations Research

mansans

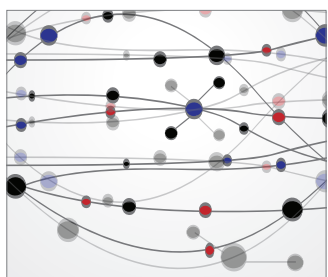

The Scientific World Journal
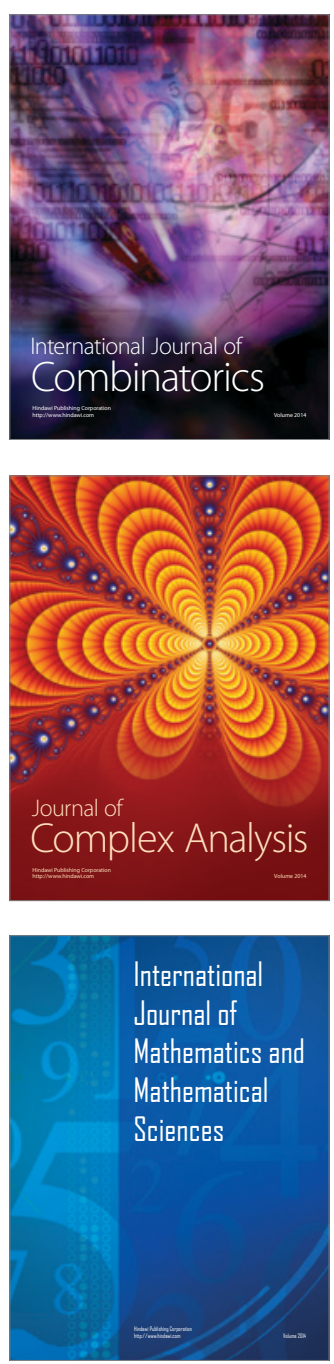
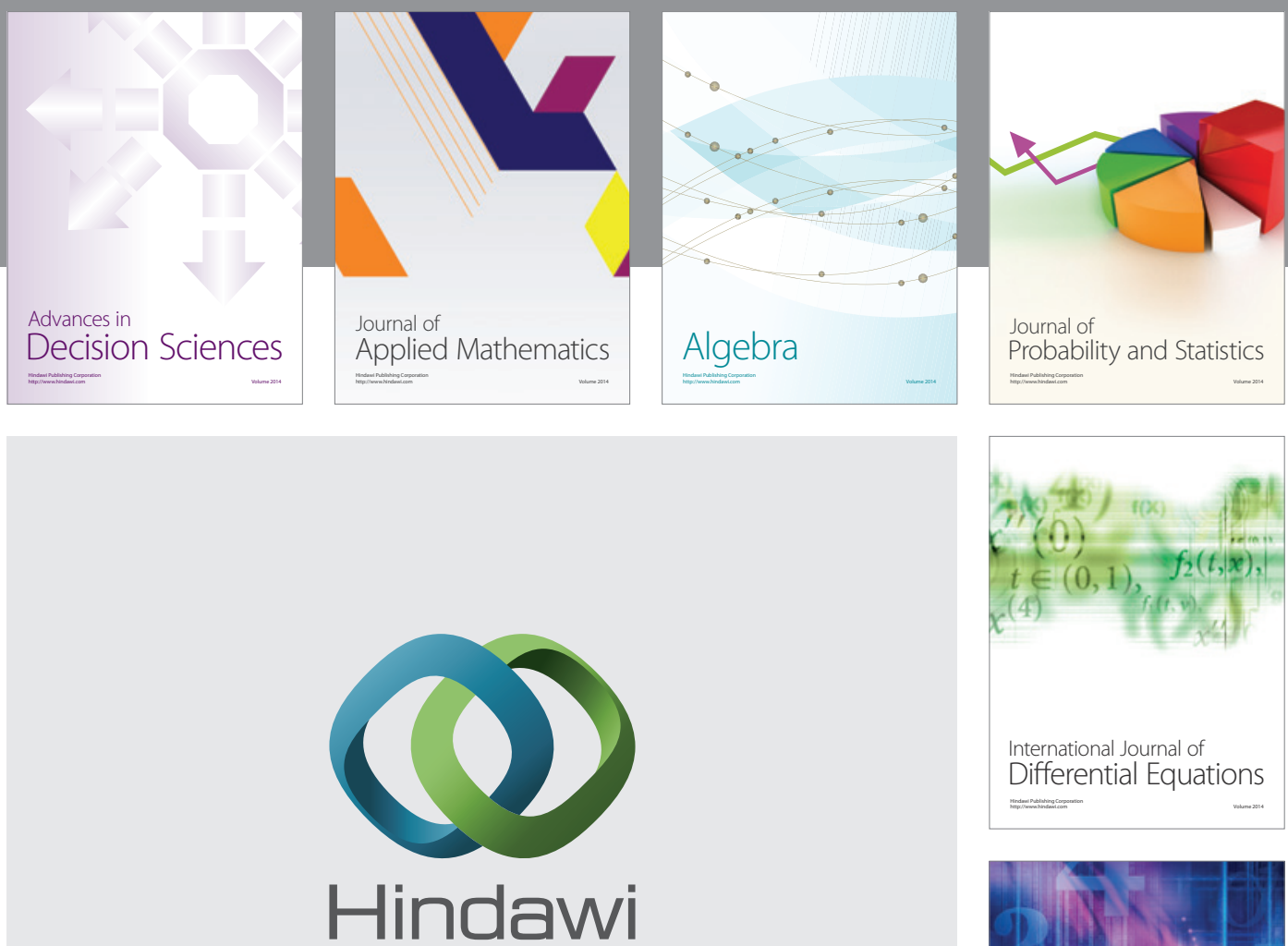

Submit your manuscripts at http://www.hindawi.com
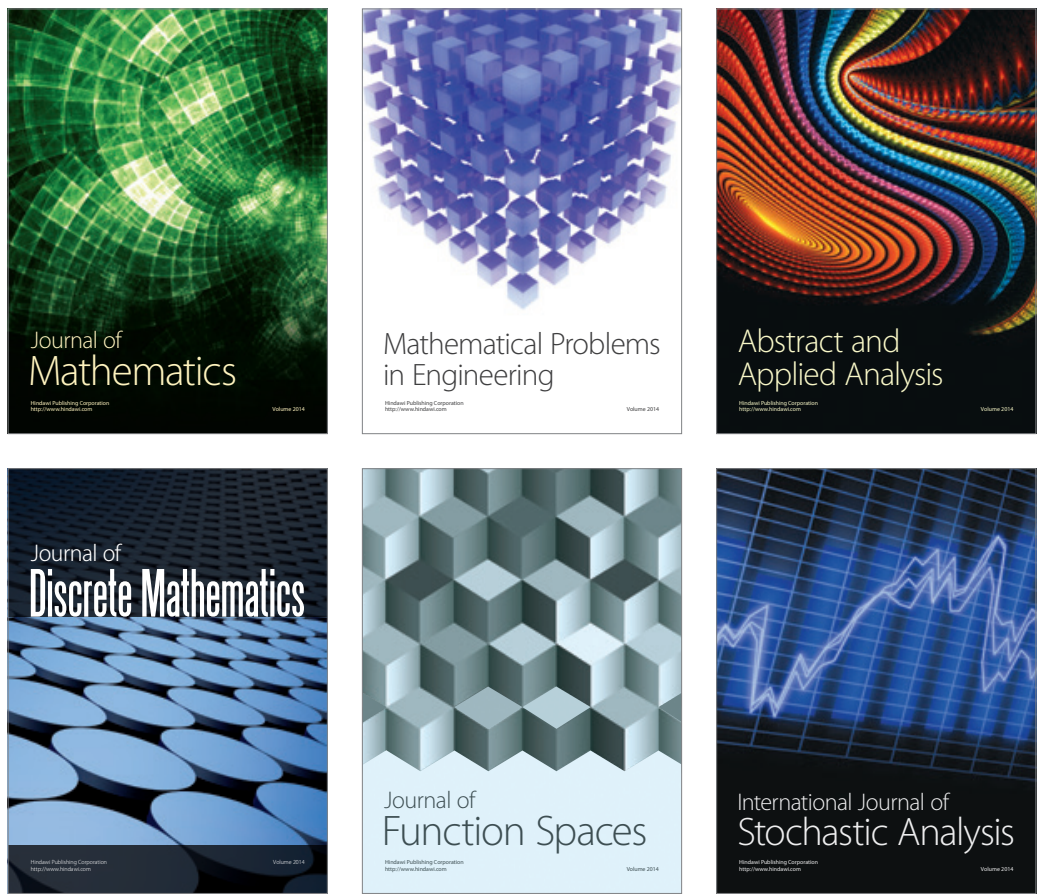

Journal of

Function Spaces

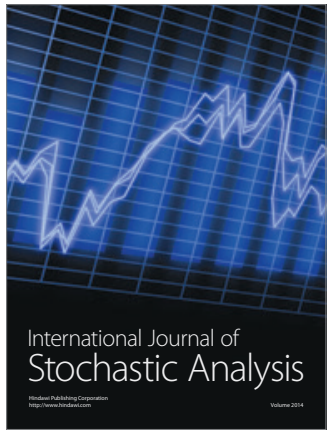

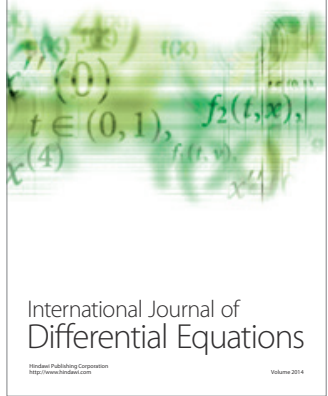
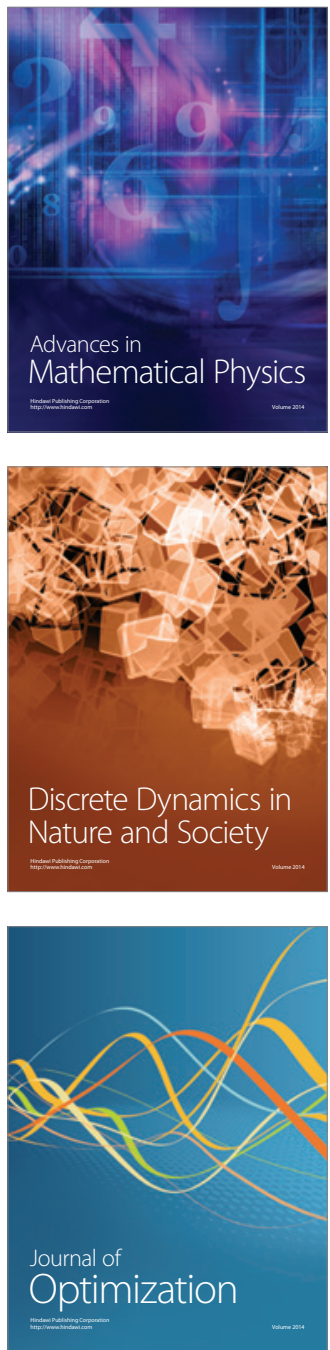